\title{
Primary CNS Lymphoma
}

\author{
Elizabeth H. Phillips • Christopher P. Fox • \\ Kate Cwynarski
}

Published online: 27 June 2014

(C) The Author(s) 2014. This article is published with open access at Springerlink.com

\begin{abstract}
Primary diffuse large B-cell lymphoma (DLBCL) of the central nervous system is an aggressive malignancy that exhibits unique biological features and characteristic clinical behaviour, with overall long-term survival rates of around 20-40\%. Clinical outcome has improved following the advent of chemoradiation protocols incorporating high-dose methotrexate in the mid1980 s, but disease relapse and adverse neurocognitive sequelae remain major clinical challenges. To address this, investigators have focused on improving drug therapy with novel cytotoxic combinations, monoclonal antibody therapy, and intensive chemotherapy consolidation approaches, in an attempt to improve disease control whilst reducing the requirement for whole-brain radiotherapy. Outcomes for patients that are older, immunocompromised, or have relapsed/refractory disease remain unsatisfactory and there is a paucity of clinical trial data to guide treatment of these groups. This review highlights recent advances in pathobiology, imaging, and clinical management of PCNSL and looks ahead to research priorities for this rare and challenging lymphoid malignancy.
\end{abstract}

Keywords CNS lymphoma · Pathobiology · Imaging · Clinical management $\cdot$ Lymphoid malignancy

\section{E. H. Phillips $\cdot$ K. Cwynarski $(\bowtie)$}

Department of Haematology, Royal Free Hospital, Pond St, London,

UK

e-mail: Kate.Cwynarski@nhs.net

C. P. Fox

Department of Clinical Haematology, Nottingham University

Hospitals NHS Trust, Nottingham, UK

\section{Introduction}

Primary central nervous system lymphoma (PCNSL) is a rare form of non-Hodgkin lymphoma (NHL) comprising $2.2 \%$ of all central nervous system (CNS) tumours [1]. It encompasses lymphoma exclusively involving the brain, spinal cord, eyes, meninges, and cranial nerves, with 90 $95 \%$ classified histologically as diffuse large B-cell lymphoma (DLBCL). The majority of PCNSL are sporadic and the incidence increases with age. A minority are attributable to immunosuppressed states, including HIV infection or iatrogenic immunosuppression following organ transplantation. In the era of effective combined antiretroviral therapy (cART), the frequency of HIV-associated PCNSL has diminished [2]. The involvement of critical sites within the CNS presents both diagnostic and therapeutic challenges, with outcomes consistently inferior to systemic DLBCL. Neurocognitive dysfunction and impaired performance status are frequent at clinical presentation, whilst histological confirmation is inherently risky and often yields small tissue biopsies. Moreover, choice of cytotoxic therapy is limited by the inability of many drugs employed for systemic NHL treatment to penetrate the blood-brain barrier (BBB) efficiently. Since the initial description of PCNSL in 1975 [3], treatment algorithms have evolved from wholebrain radiotherapy (WBRT) as a single-modality treatment towards a multi-agent, high-dose methotrexate (MTX)based, chemotherapy approach where WBRT is reserved for consolidation or for relapsed disease. Given the rarity of PCNSL, together with challenges conducting clinical trials in this patient group, data from randomised studies are scarce and the level of evidence to guide therapeutic decisions is often low. This review covers recent advances in our understanding of biological and clinical aspects of PCNSL, chiefly primary cerebral DLBCL, and potential implications for clinical practice. 


\section{Diagnosis}

The diagnosis of CNS lymphoma can be a particular challenge because of lesional response to corticosteroids and MRI features that are shared with other pathologies. The majority of PCNSL are diagnosed via stereotactic biopsy or, less commonly, by flow cytometric analysis of cerebrospinal fluid (CSF) lymphocytes. The conventional approach has been to avoid surgical resection given the risk of neurological sequelae and lack of therapeutic benefit [4]. However, a recent unplanned secondary analysis of the G-PCNSL-SG-1 trial has challenged this view, describing an apparently superior progression-free survival (PFS) for those undergoing complete or subtotal resection [5]. However, this study had a number of limitations, and independent verification in a well-designed and controlled study would be required to change practice.

Rubenstein et al. recently evaluated the utility of CXCL13 (a mediator of B-cell migration) and IL-10 as diagnostic biomarkers with the ability to discriminate CNS lymphoma from other CNS [6•]. The mean concentration of CXCL13 protein in CSF from newly diagnosed PCNSL and SCNSL was $>50$-fold higher than in CSF from patients without CNS lymphoma $\left(\mathrm{p}<1 \times 10^{-7}\right)$. The concentration of IL-10 in CSF from PCNSL and SCNSL patients was also markedly elevated compared with non-lymphoma comparators $\left(\mathrm{p}<2.3 \times 10^{-5}\right)$. Notably, for patients with PCNSL, both CXCL13 and IL10 levels below the median were associated with significantly longer PFS, although statistical independence from preexisting clinical risk scores was not shown. The positive predictive value of CXCL13 and IL-10 elevation in CSF was $95 \%$ in the identification of newly-diagnosed HIVnegative PCNSL, with an $88 \%$ negative predictive value [6•]. These interesting findings potentially offer the opportunity for CNS lymphoma diagnosis without brain biopsy, particularly where tissue biopsy is deemed high-risk or of low diagnostic yield. The precision and reproducibility of the diagnostic cut-offs, however, will need to be prospectively evaluated.

Magnetic resonance imaging (MRI) is the principal modality for the detection and monitoring of CNS lesions and recent publications have focussed on the diagnostic and prognostic role of advanced MRI techniques. Cellular density is higher and vascularity is reduced in PCNSL compared to other CNS malignancies, which is reflected in lower apparent diffusion coefficient (ADC) and relative cerebral blood flow (rCBV) values on diffusion-weighted and perfusion MRI, respectively. In support of a prior report suggesting that ADC values are predictive of outcomes in PCNSL [7], a recent study of 23 patients showed that those with baseline $\mathrm{ADC}_{\min }<384 \times$ $10^{-6} \mathrm{~mm} / \mathrm{s}$ had inferior PFS and overall survival (OS) [8]. A study by the same group reported that low baseline rCBV predicted inferior OS in a small cohort of 25 patients. Patients with both low $\mathrm{ADC}_{\min }$ and low $\mathrm{rCBV}$ had the worst outcomes with $0 \%$ OS at five years compared to $100 \%$ for those with high values for both [9]. A multi-centre phase II study failed to identify ADC as an independent prognostic factor, but numbers were small $(n=28)$ and the two-year PFS was lower in those with an ADC below the median $(57 \%$ vs. $86 \% \mathrm{p}=0.27)$ [10•].

Whole-body ${ }^{18}$ FDG PET-CT has an increased sensitivity for the detection of systemic DLBCL over conventional CT staging [11], and has an important role in the exclusion of systemic lymphoma at presentation. PCNSL lesions characteristically exhibit homogeneous, high-avidity ${ }^{18}$ FDG uptake [12], and one small study has suggested that this may assist in differentiating PCNSL from other intracranial malignancies where MRI findings are equivocal [13]. Pre-imaging corticosteroid therapy is a potential confounding factor, however, and the additional diagnostic value of ${ }^{18}$ FDG PET over modern MRI brain imaging remains poorly defined [12]. The prognostic impact of pre-treatment ${ }^{18}$ FDG PET was evaluated in a retrospective study of 42 patients by Kasenda et al., demonstrating inferior OS on multivariate analysis $(p=0.018)$ for patients with increased ${ }^{18} \mathrm{FDG}$ activity relative to cerebellar uptake at diagnosis [14], consistent with data from an earlier small $(\mathrm{n}=17)$ study [15].

\section{Molecular}

Improved characterisation of PCNSL genotype and phenotype, albeit from small studies with restricted availability of diagnostic material, has the potential to provide prognostic information and identify key molecular pathways that may serve as potential targets for novel therapeutics [16]. An activated B-cell like phenotype is typical (95\% MUM-1+, 50-80\% BCL6+, $10 \%$ CD10+), but evidence of ongoing somatic hypermutation and the preservation of an open reading frame suggests ongoing germinal centre exposure. Therefore, PCNSL does not neatly conform to either of the principal molecular profiles identified in systemic DLBCL, namely germinal centre and activated B-cell subtypes, and appears to exhibit unique transcriptional features by gene expression profiling [17]. In contrast to systemic DLBCL, high expression of BCL-2, BCL-6, and MYC by immunohistochemistry is frequent (70\% of cases studied [18]) and it has been speculated that this may contribute to the adverse prognosis of PCNSL. Recently, the only multi-centre trial to prospectively evaluate PCNSL biomarkers demonstrated that BCL-6 expression, but not MYC, correlated with inferior survival [19••]. Whilst some studies support this finding [20, 21], other retrospective analyses found that BCL- 6 overexpression correlated with superior outcomes $[22,23]$. Heterogeneous treatment approaches, sample size, and variable methodologies or cut-offs may explain these discrepant findings. 
The most frequent genomic aberration identified in PCNSL tissue is deletion of $6 \mathrm{p} 21$ involving the HLA locus (56-79\%) [24], a lesion found commonly in DLBCL arising in immuneprivileged sites [25], and represents a potential mechanism for immune escape. Deletions within the 6q22-23 region (34$50 \%$ ), which contains numerous tumour suppression genes $[20,24,26]$ and homozygous silencing/deletion of the cell cycle regulator CDKN2A (45-64 \%) [24, 27] have apparent adverse prognostic significance. The MyD-88 L265P activating mutation appears to be a common molecular aberration identified (38-50\%), but no impact on clinical outcome has been demonstrated $[21,24]$. The resultant activation of the NFKB pathway, which is also upregulated by less frequent mutations or amplification of MALT1 [28], CARD11 [29], PRDM1 [21, 30], and TBL1XR1 [24], highlights a key survival pathway and potential therapeutic target. Other dysregulated signalling pathways of potential significance include Bcell receptor signalling, with CD79B mutations in $20 \%$ [31, $32]$, and the JAK/STAT pathway $[6 \cdot, 33]$. Most of the available data, however, has emerged from relatively small studies, thus the frequency and prognostic significance of most individual genomic aberrations requires further validation, preferably in the context of prospective clinical trials.

\section{Treatment}

\section{Remission Induction}

Chemotherapy regimens incorporating HD-MTX are considered the standard of care as induction therapy for newlydiagnosed PCNSL, achieving high rates of initial response when combined with other agents. There is general consensus that MTX should be administered as a rapid infusion (24 hours) at a dose of at least $3 \mathrm{mg} / \mathrm{m}^{2}$ to maximise therapeutic CSF concentrations, at an interval of 14-21 days [34]. Higher absolute MTX exposure (area under the plasma concentrationtime curve, AUC $\left._{\text {MTX }},>980-1,100 \mu \mathrm{mol} \cdot \mathrm{h} / \mathrm{l}\right)$ correlated with superior outcomes in two post-hoc and retrospective studies $[35,36]$, but these findings have not been reproduced in all studies [37]. In patients older than 70 years, a retrospective study found that exceptionally high $\mathrm{AUC}_{\mathrm{MTX}}$ values $(>2,126 \mu \mathrm{mol} \cdot \mathrm{h} / \mathrm{l})$ inversely correlated with OS on multivariate analysis, without evidence of increased treatment toxicity [38]. Given significant inter-personal variations in MTX metabolism, an individualised dosing schedule based on pharmacokinetic analysis rather than body surface area has been suggested [39], but not prospectively validated. Recent reports that polymorphisms in key genes involved in MTX metabolism can influence outcomes and toxicity in adult lymphoma [40] and paediatric acute lymphoblastic leukaemia [41] warrant further investigation in PCNSL.
Modern protocols typically employ between four and eight cycles of HD-MTX-based therapy but comparative data on treatment duration is lacking. Historical studies of HD-MTX response have suggested minimal additional radiological response to HD-MTX beyond two cycles [42], but this phenomenon is likely to be influenced by a number of factors, including partner chemotherapy agents. In the randomised, phase II, multi-centre IELSG-20 trial, $75 \%$ of maximum responses were achieved following the first two cycles of treatment. However, for patients achieving a PR, further tumour response was observed in patients receiving combination chemotherapy (HD-MTX/Ara-C, 10/18 patients) but not HD-MTX monotherapy [43]. In a recent trial of rituximab, HD-MTX, procarbazine and vincristine (R-MPV), CR rates improved from $47 \%$ after five cycles of chemotherapy to $79 \%$ after seven [10 ]. More information on the kinetics of PCNSL response in the setting of modern immunochemotherapy protocols is required to inform the optimal duration of induction regimens.

\section{Combination Chemotherapy}

To improve on outcomes with single-agent HD-MTX, the IELSG20 trial assessed the role of combined antimetabolite therapy, with HD-MTX and cytarabine [43]. This study demonstrated a superior CR rate of $46 \%$ compared to HD-MTX alone ( $18 \%, \mathrm{p}=0.006)$, with significant improvements in PFS but not OS (three-year OS $46 \%$ vs. $32 \%, \mathrm{p}=0.07$ ). Although a relatively small study $(\mathrm{n}=79)$, it remains the first randomised trial of combination chemotherapy in PCNSL to complete accrual. Several studies have evaluated the additional value of BBB-penetrating alkylating agents (such as temozolomide, procarbazine, and thiotepa), providing non-cross resistant agents that, unlike antimetabolite chemotherapy, are also cytotoxic to cells in $\mathrm{G} 0$ of the cell cycle. Rubenstein et al. used methotrexate, temozolomide, and rituximab (MT-R) induction followed by etoposide and cytarabine (EA) consolidation in a prospective multi-centre trial of 44 patients, achieving a twoyear PFS of $57 \%$ and four-year OS of $65 \%$ [19••]. A recent multi-centre phase II trial reported promising outcomes with 52 patients treated with R-MPV induction therapy followed by reduced or standard dose WBRT $(23.4 \mathrm{~Gy}(\mathrm{n}=31)$ or $45 \mathrm{~Gy})$ and cytarabine consolidation [10•], achieving median PFS and OS of 3.3 and 6.6 years, respectively. The addition of thiotepa to HD-MTX and cytarabine was piloted in a small multicentre study $(n=20)$, with inferior results compared to the IELSG20 trial, attributed to a $50 \%$ protocol reduction in cytarabine dose ( $1 \mathrm{~g} / \mathrm{m}^{2}$ for four doses) [44], although the optimal thiotepa dose in this setting has not been ascertained. The role of thiotepa is currently being evaluated in the ongoing, randomised IELSG32 study (EudraCT number 2009012432-32). Although the outcomes of the studies discussed above appear at least comparable to those from IELSG20, meaningful comparison across studies is inherently limited 
due to differences in study design, patient characteristics, and the range of consolidation approaches. Whilst it is clear that multi-agent chemotherapy is superior to MTX alone, in the absence of further RCT evidence the optimal combination remains uncertain.

\section{Rituximab}

In contrast to the survival advantage witnessed in systemic DLBCL, the benefit of combining rituximab with chemotherapy for PCNSL remains unclear. Rituximab has limited CNS penetration with intravenous administration, achieving 0.1$4.4 \%$ of plasma concentrations [45]. Single-agent efficacy was demonstrated in 12 patients with refractory/relapsed PCNSL with radiographic response to intravenous rituximab monotherapy in $36 \%$ [46] and encouraging response rates have been achieved with rituximab $375-500 \mathrm{mg} / \mathrm{m}^{2}$ in conjunction with combination chemotherapy in single-arm trials $[8,10 \bullet, 19 \bullet, 45,47]$. Two recent studies have retrospectively compared immunochemotherapy outcomes against historical chemotherapy regimens to extrapolate the relative benefit of rituximab. Although the quality of responses appears to be improved with the addition of rituximab, no survival advantage has yet been demonstrated on multivariate analysis $[48$, 49]. Results from ongoing randomised studies (IELSG32: NCT01011920, and HOVON 105 PCNSL/ALLG NHL24 trial: EudraCT 2009-014722-42) are essential to define the role of rituximab in induction therapy for PCNSL.

\section{Intrathecal Therapy}

Detectable meningeal disease is present in $15 \%$ at diagnosis [50] and may serve as a reservoir for PCNSL cells. The presence of meningeal disease does not appear to have a prognostic impact, irrespective of whether intrathecal therapy is employed [50-52]. The additional benefit of intrathecal therapy remains unclear given the ability of HD-MTX to achieve therapeutic concentrations within the CSF [53]. Two recent non-randomised studies have suggested higher rates of early relapse with omission of intrathecal therapy in comparison with otherwise identical polychemotherapy regimens [54, 55]. Several other studies, however, have failed to demonstrate a clear improvement in response rates or OS with intrathecal therapy, leading to omission of intrathecal treatment from many current chemotherapy regimens $[51,56]$. In light of the limited ability of immunotherapy to cross the BBB, however, the role of CSF-directed therapy needs to be re-evaluated. Notably, a phase I study of intraventricular rituximab/methotrexate in 14 patients with isolated CNS lymphoma relapse, including six PCNSL patients, reported ORR and $\mathrm{CR}$ rates of $75 \%$ and $43 \%$, respectively [57•].
Consolidation Treatment

\section{WBRT}

Following introduction of HD-MTX-based chemotherapy, WBRT (36-45Gy) has continued to be employed to consolidate responses and provide more durable disease control. Delayed neurotoxicity $[58,59 \bullet]$, however, is a major limitation that is clinically evident in approximately one-third of patients, particularly with increasing age, and associated with significant morbidity and mortality. The only phase III trial thus far to complete accrual in PCNSL aimed to demonstrate that omission of consolidation WBRT after MTX-based chemotherapy resulted in non-inferior OS rates. Although outcomes suggested that WBRT can be safely omitted in selected patients achieving $\mathrm{CR}$ with induction chemotherapy, this ambitious study failed to meet its primary endpoint and major limitations in study design, amendments, and protocol adherence limit interpretation of these data [58]. A recent systematic review, which assessed outcomes of chemotherapy versus combined modality treatment using a decision-analytic model, has suggested improvements in both survival and qualityadjusted life years with consolidation WBRT for those $<60$ years only [60]. The emerging outcome data for HDTASCT consolidation for younger, sufficiently fit patients (described below), however, challenges this approach. Deferring WBRT in patients achieving CR with chemotherapy is a particularly attractive concept for those $>60$ years.

In an attempt to ameliorate the long-term neurocognitive sequelae of WBRT at standard doses, investigators have assessed the value of reduced dose WBRT (rdWBRT). Inferior outcomes have been described with a reduced consolidation WBRT dose (30.6Gy) following CHOD/BVAM induction therapy in a non-randomised comparison [61]. Morris et al. recently reported encouraging rates of disease control using 23.4Gy radiotherapy as consolidation therapy following the R-MPV protocol, with a PFS of 7.7 years for the selected subgroup $(n=31)$ achieving $C R$ with immunochemotherapy. Prospective neuropsychological evaluation demonstrated no overall cognitive decline, in 12 patients assessed 48 months after rdWBRT [10•]. The PFS for the whole cohort $(n=52)$, however, was 3.3 years in this single-arm study. Importantly, a randomised study of R-MPV versus R-MPV-rdWBRT is planned (RTOG 1114).

\section{Chemotherapy Consolidation}

The efficacy of high-dose chemotherapy and autologous stem cell transplantation (HDT-ASCT) was first demonstrated in the context of relapsed/refractory PCNSL [62] and has been subsequently studied as a consolidation approach in first-line therapy [63] in younger, fit patients. The concept of eradicating minimal residual disease by the use of myeloablative 
doses of non-cross resistant agents that efficiently penetrate the $\mathrm{BBB}$ is supported by non-comparative phase II clinical trial outcomes. Thiotepa is a small, lipophilic polyfunctional alkylator that efficiently penetrates the BBB and has been incorporated as a key agent in HDT-ASCT protocols for PCNSL. Schorb et al. retrospectively analysed outcomes from the largest reported cohort $(n=105)$ treated with thiotepa/ BCNU HDT-ASCT first-line consolidation following HDMTX-based induction. The median age was 54 and median KPS was $80 \%$. HDT-ASCT-related mortality was $2.8 \%$, and $36 \%$ of patients received additional WBRT. Outcomes were encouraging, with a five-year OS of $79 \%$ and a median PFS and OS of 85 and 121 months, respectively [64•]. On an intentionto-treat basis, long-term follow-up data was recently reported for some of these patients included in two prospective single-arm phase II trials involving HD-MTX and HD-Ara-C/thiotepa induction followed by carmustine/thiotepa-conditioned ASCT, with $(n=30)$ or without $(n=13)$ WBRT. A total of 34 of 43 patients proceeded to ASCT and median OS for the whole cohort was 104 months with a five-year OS of $70 \%$ [65]. In most studies, evidence of chemosensitivity to agents employed in induction or salvage regimens is not an absolute prerequisite to proceed to HDT-ASCT. Long-term remissions have been demonstrated in patients who failed to achieve PR with HDMTX-based induction prior to HDT-ASCT, although outcomes in this group as a whole are inferior $[64 \bullet, 66]$. No direct comparison of conditioning regimens in PCNSL has been conducted, however, earlier results using BEAM (carmustine, etoposide, cytarabine, melphalan) have been disappointing, with a PFS of 9.3 months in one study $(n=14)$ [67].

Other groups have investigated dose-intensive, nonmyeloablative, consolidation chemotherapy as an alternative strategy. A multi-centre prospective trial of MT-R induction followed by EA consolidation resulted in a median PFS of 28 months and four-year OS of $65 \%$ [19••]. Notwithstanding a relatively high rate of early disease progression, disease control using this chemotherapy consolidation strategy appears at least comparable with chemoradiation protocols. These results need further validation in an independent cohort. This strategy is potentially attractive in older or unfit patients, who are ineligible for HDT-ASCT and have higher rates of neurotoxicity with WBRT. Data from a phase II study, presented in abstract form, demonstrated the feasibility of HDAra-C ( $3 \mathrm{~g} / \mathrm{m}^{2} / \mathrm{d}$ for two days) as a consolidation approach for patients with a median age of 72 years [68]. Response-adapted consolidation based on the results of interim response assessment is another potential approach; a small retrospective study $(n=40)$ did not identify a difference in outcome with omission of WBRT or ASCT in those achieving early CR at interim assessment $(n=10)$ [69], but requires prospective validation.

Efficacy and toxicity data from a number of ongoing randomised trials in Europe and the US comparing thiotepabased HDT-ASCT with WBRT (IELSG32: NCT01011920), or non-myeloablative chemotherapy (CALGB 51101: NCT01511562), should help inform the optimal consolidation strategy in PCNSL.

\section{Salvage}

Currently, there exists no standard treatment approach for patients with relapsed and refractory PCNSL. Published data are largely restricted to small, uncontrolled, retrospective studies, in which outcomes are influenced by a number of parameters including prior therapies and response durations. With these caveats in mind, the overall response rates reported from both prospective and retrospective studies are typically $10-40 \%$. A recent retrospective analysis of MTX rechallenge described high response rates amongst 39 patients re-treated at a median of 26 months from initial diagnosis with MTX-based therapy [70]. For patients with early relapse or refractory disease, a salvage regimen employing non-crossresistant chemotherapy regimens is rational. Mappa et al. recently reported a retrospective study $(n=22)$ of salvage therapy with rituximab, ifosfamide, and etoposide for refractory $(n=11)$ or relapsed PCNSL; in this high-risk patient group the ORR was $41 \%$ with a two-year OS of $25 \%$ [71]. In studies where eligible patients have proceeded to HDTASCT, available data support the use of thiotepa-based HDTASCT in relapsed PCNSL, resulting in five-year EFS and OS rates of $37.8 \%$ and $51.4 \%$, respectively, in the largest study to date $(n=79)$ [66]. Results of WBRT as a salvage treatment are equivalent to those reported with many salvage chemotherapy regimens, with a PFS of 10-10.8 months, but are rarely durable [72, 73]. WBRT is most often employed as consolidation following salvage chemotherapy or as a palliative single-modality treatment.

Temozolomide has been evaluated in a number of retrospective [74-76] and prospective [77, 78] studies, with and without rituximab, demonstrating modest efficacy and some durable responses (ORR 14-53\%, one-year OS 31-71\%) but disappointing PFS of $<2-2.8$ months. Use of pemetrexed as an alternative antifolate agent has been reported in two small cohorts of heavily pre-treated patients with a median PFS of 5.7-5.8 months and one-year OS of $45 \%$ [79, 80] and studies addressing its efficacy are ongoing. Other agents showing limited single-agent efficacy in heavily pre-treated patients include bendamustine [81] and topotecan [82]. There is interest in assessing the efficacy of other drugs, such as lenalidomide (NCT01956695), pomalidomide (NCT01722305), temsirolimus (NCT00942747), ibrutinib, and other agents targeting B-cell signalling [16]. Given that a significant proportion of patients will not be fit for or not respond to salvage chemotherapy, there is an urgent need for the assessment of novel agents or treatment combinations in the context of clinical trials. 


\section{Older Patients}

With the median age at diagnosis of PCNSL rising above 63 years $[83,84]$ and over $20 \%$ patients older than 80 years [85], establishing optimal treatment in older patients is of paramount importance. The true incidence may be underestimated if there is reluctance to biopsy cerebral lesions in this age group, as the possible treatment options are considered to be limited. Symptoms can be misinterpreted in older patients resulting in significant treatment delays [86]. Altered metabolism of cytotoxic drugs [38] and impaired organ function [83] present additional therapeutic challenges.

Age is consistently reported as a principle adverse prognostic factor in PCNSL. Defining the 'older patient' is problematic, however, as physiological fitness does not always equate to chronological age and published studies have applied different age thresholds. In practical terms, this cohort is often defined by the ability to tolerate therapies such as WBRT ( $<60$ years) or HDT-ASCT $(<70-75$ years). The G-PCNSLSG-1 trial reported a median OS of 12.5 months in those older than 70 years (versus 26.6 months for younger patients), with a marked difference in PFS for those in CR after induction therapy (16.1 vs. 35 months $p=0.024)$ not explained by WBRT-related neurotoxicity alone [87]. The use of WBRT results in high rates of clinically significant neurotoxicity in patients over 60 years [88]. Consequently, WBRT is often avoided in those achieving CR with induction chemotherapy. WBRT offers only modest efficacy as sole therapy, with a median OS of 5-12 months [89], but may offer a palliative approach for some patients. Population-based studies, however, suggest a reluctance to use HD-MTX protocols in older patients, with 36-39\% receiving WBRT alone [85, 89]. In a cohort of 22 patients aged 80-90 years, HD-MTX was welltolerated, with only two patients requiring dose reductions and a two-year OS of $33 \%$ [86]. By contrast, a population-based study $(n=40)$ reported that $25 \%$ of patients $>60$ years were unable to tolerate more than one cycle of MTX-based chemotherapy due to renal, haematological, or pulmonary toxicity, with an additional two treatment-related deaths (5\%) [89].

A number of studies have focused on the efficacy of chemotherapy without WBRT in older patients. A randomised phase II study, published in abstract form, randomised 98 patients (median age 72 years, median KPS $70 \%$ ) to primary treatment with either MT (MTX-temozolomide) or MPV-A (methotrexate, procarbazine, vincristine, cytarabine). Efficacy endpoints favoured MPV-A (median OS 31 versus 14 months $\mathrm{p}=0.2$ ) with no increase in toxicity [68], although the study lacked the power to demonstrate statistical superiority. A phase II trial of chemoimmunotherapy with MTX, lomustine, procarbazine, and rituximab in 28 patients reported a median OS of 17.5 months [47], similar to the median OS of 15 months reported in a preceding trial without rituximab [90]. Omission of lomustine from the regimen has resulted in improved tolerability (G. Illerhaus, Stuttgart, personal communication, 2014) and formal reporting of the outcome is awaited.

\section{PCNSL in People Living With HIV}

Immunodeficiency-associated PCNSL is a distinct clinical entity characterised by Epstein Barr virus (EBV)-positivity and the presence of multifocal, often necrotic lesions on gadolinium-enhanced MRI. Incidence inversely correlates with CD4 counts and, historically, survival was strongly influenced by HIV-related comorbidities. In the cART era, the frequency of HIV-associated PCNSL has fallen whilst survival has improved [91], although outcomes remain inferior to those of immunocompetent patients [92]. Surrogate measures are often used for diagnosis, using a combination of thallium201 SPECT scanning and presence of EBV in the CSF [93, 94], therefore availability of diagnostic material for biologic studies is limited. With increasing use of intensive treatment regimens, however, histological diagnosis will have a greater influence on choice of treatment and should be obtained where feasible.

Given that HIV seropositivity is typically an exclusion criterion for prospective PCNSL studies, therapy has been empirically adopted from treatment of PCNSL in immunocompetent patients, akin to the paradigm of systemic lymphoma therapy [95]. Case reports of isolated responses to cART alone are few [96] and subject to selection bias, therefore, it is not recommended as the sole treatment modality. Use of either WBRT or chemotherapy independently reduced the risk of death in a large retrospective HIV-PCNSL cohort [91]. HDMTX-based regimens have been tolerated in immunodeficient patients, but trials in the cART era are lacking [97] and data is particularly scarce on patients receiving combination chemotherapy $[92,98]$. There is an isolated case report of successful ASCT [99]. Despite this, in large population-based studies, reasonable survival rates of $54 \%$ at one year [100] and $22.8 \%$ at five years [101] were reported.

\section{Post-Treatment Evaluation and Monitoring}

Treatment-related neurotoxicity occurring months to years following completion of therapy is frequently a progressive condition that can be disabling, even fatal, in some patients. The risk of neurotoxicity correlates with the dose of WBRT and increasing age [102, 103]. Whether combination chemotherapy confers additive risk of neurotoxicity is not yet clear, largely due to the paucity of prospective psychometric assessment in many trials. A standardised battery of neuropsychological and quality of life assessments have been published and are being incorporated into ongoing prospective studies [103]. Recently, Doolittle et al. [59•] reported on the long-term cognitive outcomes in 80 PCNSL survivors who achieved CR 
with HD-MTX-based protocols, of which $19 \%$ incorporated WBRT at a dose of $45 \mathrm{~Gy}$ or greater. At a median follow-up of 5.5 years, $47 \%$ of patients treated with chemoradiation protocols demonstrated impairment in multiple cognitive domains, compared to $9-16 \%$ receiving chemotherapy alone $(\mathrm{p}=0.0237)$. Correa et al. presented cognitive assessment on 50 patients, 24 of whom received WBRT plus chemotherapy and 26 who received chemotherapy alone. Quality of life was lower in WBRT patients, with significant reductions throughout all cognitive domains compared to age-matched controls [104]. Interpretation of both cross-sectional studies, however, is limited by lack of formal assessment of pre- and posttreatment cognitive status. Almost all patients with delayed neurotoxicity have marked white matter changes on MRI [102], and the extent of white matter changes has been shown to be correlated inversely with cognitive function in some studies [59•] but not others [104]. This is a common posttreatment finding in PCNSL, however, and is not an adequate surrogate for formal psychometric assessment.

A large, single-centre retrospective $(n=209)$ of PCNSL patients monitored with post-treatment brain imaging every 4-6 months demonstrated that, of 124 patients in CR, $80 \%$ of relapses that occurred were symptomatic and detected between surveillance scans [105•]. Given the incidence of late relapses and lack of evidence for early salvage treatment, there is no clear indication for surveillance imaging outside of clinical trials.

Relatively few studies have reported survival rates beyond five years, with varying incidence of late relapse and limited evidence for emergence of a survival plateau. Relapses can occur more than 10 years after initial diagnosis, with evidence of clonal persistence [106]. In a single-centre retrospective cohort, of those achieving CR with induction therapy ( $\mathrm{n}=$ 268), 10 out of 48 patients in ongoing remission at five years subsequently relapsed (20.8 \%) [106]. Prospective follow-up of 41 patients treated with chemoradiotherapy at a median of 12 years has suggested the emergence of a survival plateau with a 10 -year OS of $24 \%$ [107]. Long-term follow-up of patients in two trials involving ASCT consolidation $(n=43)$ reported that $50 \%$ of relapses $(6 / 12)$ occurred more than five years post-treatment [65] and emphasises the need for longterm follow-up in PCNSL survivors to fully evaluate treatment efficacy.

\section{Conclusions}

Information from randomised clinical trials is essential to further improve the management of PCNSL and answer critical questions such as the optimal induction chemotherapy and consolidation strategy. Correlative biological studies in the context of prospective trials will refine our knowledge of PCNSL biology and potentially enable identification of those with poor-risk disease at diagnosis, for whom intensification of treatment and/or novel therapeutics may be of benefit. Focussing on neurocognitive outcomes and long-term follow-up of survivors will allow refinement of the balance between treatment toxicity and cure.

\section{Compliance with Ethics Guidelines}

Conflict of Interest Dr. Elizabeth H. Phillips declares no potential conflicts of interest.

Dr. Christopher Fox is a consultant for Roche UK. Dr. Fox received honoraria and travel/accommodations expenses covered or reimbursed by Roche UK.

Dr. Kate Cwynarski received honoraria from Roche and Adienne, and travel/accommodations expenses covered or reimbursed by Roche.

Human and Animal Rights and Informed Consent This article does not contain any studies with human or animal subjects performed by any of the authors.

Open Access This article is distributed under the terms of the Creative Commons Attribution License which permits any use, distribution, and reproduction in any medium, provided the original author(s) and the source are credited.

\section{References}

Papers of particular interest, published recently, have been highlighted as:

- Of importance

•. Of major importance

1. Dolecek TA, Propp JM, Stroup NE, Kruchko C. CBTRUS statistical report: Primary brain and central nervous system tumors diagnosed in the United States in 2005-2009. Neuro Oncol. 2012;14.

2. Shiels MS, Pfeiffer RM, Hall HI, Li J, Goedert JJ, Morton LM, et al. Proportions of Kaposi sarcoma, selected non-Hodgkin lymphomas, and cervical cancer in the United States occurring in persons with AIDS, 1980-2007. JAMA. 2011;305:1450-9.

3. Henry J, Heffner RJ, Dillard S, Earle K, Davis R. Primary malignant lymphomas of the central nervous system. Cancer. 1974;34: 1293-302.

4. Bellinzona M, Roser F, Ostertag H, Gaab RM, Saini M. Surgical removal of primary central nervous system lymphomas (PCNSL) presenting as space occupying lesions: a series of 33 cases. Eur J Surg Oncol. 2005;31:100-5.

5. Weller M, Martus P, Roth P, Thiel E, Korfel A. Surgery for primary CNS lymphoma? Challenging a paradigm. Neuro Oncol. 2012;14:1481-4.

6. Rubenstein JL, Wong VS, Kadoch C, Gao HX, Barajas R, Chen L, et al. CXCL13 plus interleukin 10 is highly specific for the diagnosis of CNS lymphoma. Blood. 2013;121:4740-8. Assessed CXCL13 and IL-10 as potential CSF biomarkers of PCNSL on a large cohort of patients. Findings were integrated with biological data demonstrating effects on chemotaxis and activation of potential downstream signalling pathways.

7. Barajas RF, Rubenstein JL, Chang JS, Hwang J, Cha S. Diffusionweighted MR imaging derived apparent diffusion coefficient is predictive of clinical outcome in primary central nervous system lymphoma. Am J Neuroradiol. 2010;31:60-6. 
8. Wieduwilt MJ, Valles F, Issa S, Behler CM, Hwang J, McDermott $\mathrm{M}$, et al. Immunochemotherapy with intensive consolidation for primary CNS lymphoma: a pilot study and prognostic assessment by diffusion-weighted MRI. Clin Cancer Res. 2012;18:1146-55.

9. Valles FE, Perez-Valles CL, Regalado S, Barajas RF, Rubenstein JL, Cha S. Combined diffusion and perfusion MR imaging as biomarkers of prognosis in immunocompetent patients with primary central nervous system lymphoma. AJNR Am J Neuroradiol. 2013;34:35-40.

10. Morris PG, Correa DD, Yahalom J, Raizer JJ, Schiff D, Grant B, et al. Rituximab, methotrexate, procarbazine, and vincristine followed by consolidation reduced-dose whole-brain radiotherapy and cytarabine in newly diagnosed primary CNS lymphoma: final results and long-term outcome. J Clin Oncol. 2013;31:3971-9. Multi-centre prospective single-arm trial assessing the role of reduced-dose radiotherapy for patients in CR following induction chemotherapy, in an attempt to reduce late neurotoxic effects. Treatment-related and neurocognitive outcomes were encouraging.

11. Mohile NA, Deangelis LM, Abrey LE. The utility of body FDG PET in staging primary central nervous system lymphoma. Neuro Oncol. 2008;10:223-8.

12. Kawai N, Miyake K, Yamamoto Y, Nishiyama Y, Tamiya T. 18FFDG PET in the diagnosis and treatment of primary central nervous system lymphoma. Biomed Res Int. 2013;2013:247152.

13. Makino K, Hirai T, Nakamura H, Murakami R, Kitajima M, Shigematsu Y, et al. Does adding FDG-PET to MRI improve the differentiation between primary cerebral lymphoma and glioblastoma? Observer performance study. Ann Nucl Med. 2011;25:432-8.

14. Kasenda B, Haug V, Schorb E, Fritsch K, Finke J, Mix M, et al. 18 F-FDG PET is an independent outcome predictor in primary central nervous system lymphoma. J Nucl Med. 2013;54:184-91.

15. Kawai N, Zhen H-N, Miyake K, Yamamaoto Y, Nishiyama Y, Tamiya T. Prognostic value of pretreatment 18F-FDG PET in patients with primary central nervous system lymphoma: SUVbased assessment. J Neurooncol. 2010;100:225-32.

16. Ponzoni M, Issa S, Batchelor TT, Rubenstein JL. Beyond highdose methotrexate and brain radiotherapy: novel targets and agents for primary CNS lymphoma. Ann Oncol. 2014;25:316-22.

17. Tun HW, Personett D, Baskerville KA, Menke DM, Jaeckle KA, Kreinest $\mathrm{P}$, et al. Pathway analysis of primary central nervous system lymphoma. Blood. 2008;111:3200-10.

18. Brunn A, Nagel I, Montesinos-Rongen M, Klapper W, Vater I, Paulus W, et al. Frequent triple-hit expression of MYC, BCL2, and BCL6 in primary lymphoma of the central nervous system and absence of a favorable MYC(low)BCL2 (low) subgroup may underlie the inferior prognosis as compared to systemic diffuse large B cell lymphom. Acta Neuropathol. 2013;126:603-5.

19.• Rubenstein JL, Hsi ED, Johnson JL, Jung SH, Nakashima MO, Grant B, et al. Intensive chemotherapy and immunotherapy in patients with newly diagnosed primary CNS lymphoma: CALGB 50202 (Alliance 50202). J Clin Oncol. 2013;31:30618. Prospectively correlated biological data with outcomes. Provided evidence in a multi-centre single-arm trial that reasonable response rates and outcomes can be achieved with intensive consolidation chemotherapy, without subsequent WBRT or ASCT.

20. Cady FM, O'Neill BP, Law ME, Decker PA, Kurtz DM, Giannini C, et al. Del(6)(q22) and BCL6 rearrangements in primary CNS lymphoma are indicators of an aggressive clinical course. J Clin Oncol. 2008;26:4814-9.

21. Montesinos-Rongen M, Godlewska E, Brunn A, Wiestler OD, Siebert R, Deckert M. Activating L265P mutations of the MYD88 gene are common in primary central nervous system lymphoma. Acta Neuropathol. 2011;122:791-2.

22. Braaten KM, Betensky RA, de Leval L, Okada Y, Hochberg FH, Louis DN, et al. BCL-6 expression predicts improved survival in patients with primary central nervous system lymphoma. Clin Cancer Res. 2003;9:1063-9.

23. Levy O, DeAngelis LM, Filippa DA, Panageas KS, Abrey LE. Bcl-6 predicts improved prognosis in primary central nervous system lymphoma. Cancer. 2008;112:151-6.

24. Gonzalez-Aguilar A, Idbaih A, Boisselier B, Habbita N, Rossetto $\mathrm{M}$, Laurenge A, et al. Recurrent mutations of MYD88 and TBL1XR1 in primary central nervous system lymphomas. Clin Cancer Res. 2012;18:5203-11.

25. Booman M, Szuhai K, Rosenwald A, Hartmann E, KluinNelemans HC, De Jong D, et al. Genomic alterations and gene expression in primary diffuse large B-cell lymphomas of immuneprivileged sites: the importance of apoptosis and immunomodulatory pathways. J Pathol. 2008;216:209-17.

26. McPhail ER, Law ME, Decker PA, O'Neill BP. Influence of 6q2223 on overall survival in primary central nervous system lymphoma. Analysis of North Central Cancer Treatment Group trials 86 72 52, 937351 and 9673 51. Br J Haematol. 2011;154:146-50.

27. Hayashi Y, Iwato M, Arakawa Y, Fujisawa H, Thoma Y, Hasegawa M, et al. Homozygous deletion of INK4a/ARF genes and overexpression of bcl-2 in relation with poor prognosis in immunocompetent patients with primary central nervous system lymphoma of the diffuse large B-cell type. J Neurooncol. 2001;55:51-8.

28. Schwindt H, Vater I, Kreuz M, Montesinos-Rongen M, Brunn A, Richter J, et al. Chromosomal imbalances and partial uniparental disomies in primary central nervous system lymphoma. Leukemia. 2009;23:1875-84.

29. Montesinos-Rongen M, Schmitz R, Brunn A, Gesk S, Richter J, Hong K, et al. Mutations of CARD11 but not TNFAIP3 may activate the NF-??B pathway in primary CNS lymphoma. Acta Neuropathol. 2010;120:529-35.

30. Courts C, Montesinos-Rongen M, Brunn A, Bug S, Siemer D, Hans V, et al. Recurrent inactivation of the PRDM1 gene in primary central nervous system lymphoma. J Neuropathol Exp Neurol. 2008;67:720-7.

31. Kraan W, Horlings HM, van Keimpema M, Schilder-Tol EJM, Oud MECM, Scheepstra C, et al. High prevalence of oncogenic MYD88 and CD79B mutations in diffuse large B-cell lymphomas presenting at immune-privileged sites. Blood Cancer J. 2013;3:e139.

32. Montesinos-Rongen M, Schafer E, Siebert R, Deckert M. Genes regulating the $\mathrm{B}$ cell receptor pathway are recurrently mutated in primary central nervous system lymphoma. Acta Neuropathol. 2012;124:905-6.

33. Sung CO, Kim SC, Karnan S, Karube K, Shin HJ, Nam DH, et al. Genomic profiling combined with gene expression profiling in primary central nervous system lymphoma. Blood. 2011;117: 1291-300.

34. Joerger M, Huitema ADR, Illerhaus G, Ferreri AJM. Rational administration schedule for high-dose methotrexate in patients with primary central nervous system lymphoma. Leuk Lymphoma. 2012;53:1867-75.

35. Ferreri AJM, Guerra E, Regazzi M, Pasini F, Ambrosetti A, Pivnik A, et al. Area under the curve of methotrexate and creatinine clearance are outcome-determining factors in primary CNS lymphomas. Br J Cancer. 2004;90:353-8.

36. Joerger M, Huitema ADR, Krähenbühl S, Schellens JHM, Cerny $\mathrm{T}$, Reni M, et al. Methotrexate area under the curve is an important outcome predictor in patients with primary CNS lymphoma: a pharmacokinetic-pharmacodynamic analysis from the IELSG no. 20 trial. Br J Cancer. 2010;102:673-7.

37. Morris PG, Abrey LE, Reiner AS, Wu N, Panageas KS, Seko BS, et al. Methotrexate area under the curve as a prognostic factor in primary central nervous system lymphoma treated with immunochemoradiotherapy. Leuk Lymphoma. 2011;52:1891-7.

38. Kasenda B, Rehberg M, Thürmann P, Franzem M, Veelken H, Fritsch K, et al. The prognostic value of serum methotrexate area 
under curve in elderly primary CNS lymphoma patients. Ann Hematol. 2012;91:1257-64.

39. Joerger M, Ferreri AJM, Krähenbühl S, Schellens JHM, Cerny T, Zucca E, et al. Dosing algorithm to target a predefined AUC in patients with primary central nervous system lymphoma receiving high dose methotrexate. Br J Clin Pharmacol. 2012;73:240-7.

40. Chiusolo P, Giammarco S, Bellesi S, Metafuni E, Piccirillo N, De Ritis D, et al. The role of MTHFR and RFC1 polymorphisms on toxicity and outcome of adult patients with hematological malignancies treated with high-dose methotrexate followed by leucovorin rescue. Cancer Chemother Pharmacol. 2012;69:691-6.

41. Radtke S, Zolk O, Renner B, Paulides M, Zimmermann M, Möricke A, et al. Germline genetic variations in methotrexate candidate genes are associated with pharmacokinetics, toxicity, and outcome in childhood acute lymphoblastic leukemia. Blood. 2013;121:5145-53.

42. Hiraga S, Arita N, Ohnishi T, Kohmura E, Yamamoto K, Oku Y, et al. Rapid infusion of high-dose methotrexate resulting in enhanced penetration into cerebrospinal fluid and intensified tumor response in primary central nervous system lymphomas. J Neurosurg. 1999;91:221-30.

43. Ferreri AJ, Reni M, Foppoli M, Martelli M, Pangalis GA, Frezzato M, et al. High-dose cytarabine plus high-dose methotrexate versus high-dose methotrexate alone in patients with primary CNS lymphoma: a randomised phase 2 trial. Lancet. 2009;374:1512-20.

44. Ferreri AJM, Licata G, Foppoli M, Corazzelli G, Zucca E, Stelitano C, et al. Clinical relevance of the dose of cytarabine in the upfront treatment of primary CNS lymphomas with methotrexate-cytarabine combination. Oncologist. 2011;16:33641.

45. Shah GD, Yahalom J, Correa DD, Lai RK, Raizer JJ, Schiff D, et al. Combined immunochemotherapy with reduced whole-brain radiotherapy for newly diagnosed primary CNS lymphoma. J Clin Oncol. 2007;25:4730-5.

46. Batchelor TT, Grossman SA, Mikkelsen T, Ye X, Desideri S, Lesser GJ. Rituximab monotherapy for patients with recurrent primary CNS lymphoma. Neurology. 2011/03/09 ed. 2011;76: 929-30.

47. Fritsch K, Kasenda B, Hader C, Nikkhah G, Prinz M, Haug V, et al. Immunochemotherapy with rituximab, methotrexate, procarbazine, and lomustine for primary CNS lymphoma (PCNSL) in the elderly. Ann Oncol. 2011;22:2080-5.

48. Birnbaum T, Stadler EA, Von Baumgarten L, Straube A. Rituximab significantly improves complete response rate in patients with primary CNS lymphoma. J Neurooncol. 2012;109: 285-91.

49. Gregory G, Arumugaswamy A, Leung T, Chan KL, Abikhair M, Tam C, et al. Rituximab is associated with improved survival for aggressive B cell CNS lymphoma. Neuro Oncol. 2013;15:106873.

50. Korfel A, Weller M, Martus P, Roth P, Klasen HA, Roeth A, et al. Prognostic impact of meningeal dissemination in primary CNS lymphoma (PCNSL): experience from the G-PCNSL-SG1 trial. Ann Oncol. 2012;23:2374-80.

51. Khan RB, Shi W, Thaler HT, DeAngelis LM, Abrey LE. Is intrathecal methotrexate necessary in the treatment of primary CNS lymphoma? J Neurooncol. 2002;58:175-8.

52. Taylor JW, Flanagan EP, O'Neill BP, Siegal T, Omuro A, DeAngelis L, et al. Primary leptomeningeal lymphoma: international primary CNS lymphoma collaborative group report. Neurology. 2013;81:1690-6.

53. Glantz MJ, Cole BF, Recht L, Akerley W, Mills P, Saris S, et al. High-dose intravenous methotrexate for patients with nonleukemic leptomeningeal cancer: is intrathecal chemotherapy necessary? J Clin Oncol. 1998;16:1561-7.
54. Pels H, Juergens A, Glasmacher A, Schulz H, Engert A, Linnebank M, et al. Early relapses in primary CNS lymphoma after response to polychemotherapy without intraventricular treatment: results of a phase II study. J Neurooncol. 2009;91:299-305.

55. Sierra Del Rio M, Ricard D, Houillier C, Navarro S, GonzalezAguilar A, Idbaih A, et al. Prophylactic intrathecal chemotherapy in primary CNS lymphoma. J Neurooncol. 2012;106:143-6.

56. Ferreri AJM, Reni M, Pasini F, Calderoni A, Tirelli U, Pivnik A, et al. A multicenter study of treatment of primary CNS lymphoma. Neurology. 2002;58:1513-20.

57. Rubenstein JL, Li J, Chen L, Advani R, Drappatz J, Gerstner E, et al. Multicenter phase 1 trial of intraventricular immunochemotherapy in recurrent CNS lymphoma. Blood. 2013;121:745-51. Demonstrated the efficacy intraventricular rituximab in the relapse setting without systemic chemotherapy. Depending on the efficacy of intravenous rituximab in ongoing randomised trials, it may warrant re-evaluation of the role of intrathecal treatment in PCNSL.

58. Thiel E, Korfel A, Martus P, Kanz L, Griesinger F, Rauch M, et al. High-dose methotrexate with or without whole brain radiotherapy for primary CNS lymphoma (G-PCNSL-SG-1): a phase 3, randomised, non-inferiority trial. Lancet Oncol. 2010;11:103647.

59. Doolittle ND, Korfel A, Lubow MA, Schorb E, Schlegel U, Rogowski S, et al. Long-term cognitive function, neuroimaging, and quality of life in primary CNS lymphoma. Neurology. 2013;81:84-92. The most comprehensive formal assessment of long-term neurocognitive outcomes to date, comparing neurocognitive late effects in those receiving a variety of treatment modalities and demonstrating the extent of neurocognitive impairment throughout multiple domains attributable to WBRT.

60. Prica A, Chan K, Cheung MC. Combined modality therapy versus chemotherapy alone as an induction regimen for primary central nervous system lymphoma: a decision analysis. Br J Haematol. 2012;158:600-7.

61. Bessell EM, López-Guillermo A, Villá S, Verger E, Nomdedeu B, Petit J, et al. Importance of radiotherapy in the outcome of patients with primary CNS lymphoma: an analysis of the CHOD/BVAM regimen followed by two different radiotherapy treatments. J Clin Oncol. 2002;20:231-6.

62. Soussain C, Hoang-Xuan K, Taillandier L, Fourme E, Choquet S, Witz F, et al. Intensive chemotherapy followed by hematopoietic stem-cell rescue for refractory and recurrent primary CNS and intraocular lymphoma: Société Française de Greffe de Moëlle Osseuse-Thérapie Cellulaire. J Clin Oncol. 2008;26:2512-8.

63. Illerhaus G, Marks R, Ihorst G, Guttenberger R, Ostertag C, Derigs $G$, et al. High-dose chemotherapy with autologous stem-cell transplantation and hyperfractionated radiotherapy as first-line treatment of primary CNS lymphoma. J Clin Oncol. 2006;24:3865-70.

64. Schorb E, Kasenda B, Atta J, Kaun S, Morgner A, Hess G, et al. Prognosis of patients with primary central nervous system lymphoma after high-dose chemotherapy followed by autologous stem cell transplantation. Haematologica. 2013;98:765-70. Largest retrospective study of first-line ASCT consolidation with long-term follow-up to date demonstrating very good long-term outcomes.

65. Kasenda B, Schorb E, Fritsch K, Finke J, Illerhaus G. Prognosis after high-dose chemotherapy followed by autologous stem-cell transplantation as first-line treatment in primary CNS lymphomaa long-term follow-up study. Ann Oncol. 2012;23:2670-5.

66. Soussain C, Choquet S, Fourme E, Delgadillo D, Bouabdallah K, Ghesquières $\mathrm{H}$, et al. Intensive chemotherapy with thiotepa, busulfan and cyclophosphamide and hematopoietic stem cell rescue in relapsed or refractory primary central nervous system 
lymphoma and intraocular lymphoma: a retrospective study of 79 cases. Haematologica. 2012;97:1751-6.

67. Abrey LE, Moskowitz CH, Mason WP, Crump M, Stewart D, Forsyth $\mathrm{P}$, et al. Intensive methotrexate and cytarabine followed by high-dose chemotherapy with autologous stem-cell rescue in patients with newly diagnosed primary CNS lymphoma: an intentto-treat analysis. J Clin Oncol. 2003;21:4151-6.

68. Omuro AMP, Chinot $\mathrm{O}$, Taillandier $\mathrm{L}$, Ghesquières $\mathrm{H}$, Soussain $\mathrm{C}$, Delwail V, et al. Multicenter randomized phase II trial of methotrexate (MTX) and temozolomide (TMZ) versus MTX, procarbazine, vincristine, and cytarabine for primary CNS lymphoma (PCNSL) in the elderly: an Anocef and Goelams Intergroup study. J Clin Oncol. 2013;31:2032.

69. Kim YR, Kim SH, Chang JH, Suh CO, Kim SJ, Kim Y, et al. Early response to high-dose methotrexate, vincristine, and procarbazine chemotherapy-adapted strategy for primary CNS lymphoma: no consolidation therapy for patients achieving early complete response. Ann Hematol. 2014;93:211-9.

70. Pentsova E, DeAngelis LM, Omuro A. Methotrexate re-challenge for recurrent primary central nervous system lymphoma. J Neurooncol. 2014;117:161-5.

71. Mappa S, Marturano E, Licata G, Frezzato M, Frungillo N, Ilariucci F, et al. Salvage chemoimmunotherapy with rituximab, ifosfamide and etoposide (R-IE regimen) in patients with primary CNS lymphoma relapsed or refractory to high-dose methotrexatebased chemotherapy. Hematol Oncol. 2013;31:143-50.

72. Nguyen PL, Chakravarti A, Finkelstein DM, Hochberg FH, Batchelor TT, Loeffler JS. Results of whole-brain radiation as salvage of methotrexate failure for immunocompetent patients with primary CNS lymphoma. J Clin Oncol. 2005;23:1507-13.

73. Hottinger AF, Deangelis LM, Yahalom J, Abrey LE. Salvage whole brain radiotherapy for recurrent or refractory primary CNS lymphoma. Neurology. 2007;69:1178-82.

74. Enting RH, Demopoulos A, DeAngelis LM, Abrey LE. Salvage therapy for primary CNS lymphoma with a combination of rituximab and temozolomide. Neurology. 2004;63:901-3.

75. Makino K, Nakamura H, Hide TI, Kuratsu JI. Salvage treatment with temozolomide in refractory or relapsed primary central nervous system lymphoma and assessment of the MGMT status. J Neurooncol. 2012;106:155-60.

76. Wong SF, Gan HK, Cher L. A single centre study of the treatment of relapsed primary central nervous system lymphoma (PCNSL) with single agent temozolomide. J Clin Neurosci. 2012;19:1501-5.

77. Reni M, Zaja F, Mason W, Perry J, Mazza E, Spina M, et al. Temozolomide as salvage treatment in primary brain lymphomas. Br J Cancer. 2007;96:864-7.

78. Nayak L, Abrey LE, Drappatz J, Gilbert MR, Reardon DA, Wen PY, et al. Multicenter phase II study of rituximab and temozolomide in recurrent primary central nervous system lymphoma. Leuk Lymphoma. 2013;54:58-61.

79. Raizer JJ, Rademaker A, Evens AM, Rice L, Schwartz M, Chandler JP, et al. Pemetrexed in the treatment of relapsed/ refractory primary central nervous system lymphoma. Cancer. 2012;118:3743-8.

80. Zhang J-P, Lee EQ, Nayak L, Doherty L, Kesari S, Muzikansky A, et al. Retrospective study of pemetrexed as salvage therapy for central nervous system lymphoma. J Neurooncol. 2013;115:71-7.

81. Chamberlain MC. Salvage therapy with bendamustine for methotrexate refractory recurrent primary CNS lymphoma: a retrospective case series. J. Neurooncol. 2014;1-8.

82. Fischer L, Thiel E, Klasen H, Birkmann J, Jahnke K, Martus P, et al. Prospective trial on topotecan salvage therapy in primary CNS lymphoma. Ann Oncol. 2006;17:1141-5.

83. Bessell EM, Dickinson P, Dickinson S, Salmon J. Increasing age at diagnosis and worsening renal function in patients with primary central nervous system lymphoma. J Neurooncol. 2011;104:191-3.
84. Shibamoto Y, Sumi M, Onodera S, Matsushita H, Sugie C, Tamaki $\mathrm{Y}$, et al. Primary CNS lymphoma treated with radiotherapy in Japan: a survey of patients treated in 2005-2009 and a comparison with those treated in 1985-2004. Int J Clin Oncol. 2013;1-9.

85. Panageas KS, Elkin EB, Ben-Porat L, DeAngelis LM, Abrey LE. Patterns of treatment in older adults with primary central nervous system lymphoma. Cancer. 2007;110:1338-44.

86. Welch MR, Omuro A, Deangelis LM. Outcomes of the oldest patients with primary CNS lymphoma treated at Memorial SloanKettering Cancer Center. Neuro Oncol. 2012;14:1304-11.

87. Roth P, Martus P, Kiewe P, Möhle R, Klasen H, Rauch M, et al. Outcome of elderly patients with primary CNS lymphoma in the G-PCNSL-SG-1 trial. Neurology. 2012;79:890-6.

88. Abrey LE, DeAngelis LM, Yahalom J. Long-term survival in primary CNS lymphoma. J Clin Oncol. 1998;16:859-63.

89. Schuurmans M, Bromberg JEC, Doorduijn J, Poortmans P, Taphoorn MJB, Seute T, et al. Primary central nervous system lymphoma in the elderly: a multicentre retrospective analysis. Br J Haematol. 2010;151:179-84.

90. Illerhaus G, Marks R, Müller F, Ihorst G, Feuerhake F, Deckert M, et al. High-dose methotrexate combined with procarbazine and CCNU for primary CNS lymphoma in the elderly: results of a prospective pilot and phase II study. Ann Oncol. 2009;20:319-25.

91. Uldrick TS, Pipkin S, Scheer S, Hessol NA. Factors associated with survival among patients with AIDS-related primary central nervous system lymphoma. AIDS. 2013;28:397-405.

92. Bayraktar S, Bayraktar UD, Ramos JC, Stefanovic A, Lossos IS. Primary CNS lymphoma in HIV positive and negative patients: comparison of clinical characteristics, outcome and prognostic factors. J Neurooncol. 2011;101:257-65.

93. Antinori A, De Rossi G, Ammassari A, Cingolani A, Murri R, Di Giuda D, et al. Value of combined approach with thallium-201 single-photon emission computed tomography and Epstein-Barr virus DNA polymerase chain reaction in CSF for the diagnosis of AIDS-related primary CNS lymphoma. J Clin Oncol. 1999;17: 554-60.

94. Yanagisawa K, Tanuma J, Hagiwara S, Gatanaga H, Kikuchi Y, Oka S. Epstein-barr viral load in cerebrospinal fluid as a diagnostic marker of central nervous system involvement of AIDS-related lymphoma. Intern Med. 2013;52:955-9.

95. Barta SK, Xue X, Wang D, Tamari R, Lee JY, Mounier N, et al. Treatment factors affecting outcomes in HIV-associated nonHodgkin lymphomas: a pooled analysis of 1546 patients. Blood. 2013;122:3251-62.

96. Travi G, Ferreri AJM, Cinque P, Gerevini S, Ponzoni M, Terreni $\mathrm{M}$, et al. Long-term remission of HIV-associated primary CNS lymphoma achieved with highly active antiretroviral therapy alone. Infection. 2012;30:119-21.

97. González-Aguilar A, Soto-Hernández JL. The management of primary central nervous system lymphoma related to AIDS in the HAART era. Curr Opin Oncol. 2011;23:648-53.

98. Nagai H, Odawara T, Ajisawa A, Hagiwara S, Watanabe T, Uehira $\mathrm{T}$, et al. Whole brain radiation alone produces favourable outcomes for AIDS-related primary central nervous system lymphoma in the HAART era. Eur J Haematol. 2010;84:499-505.

99. Wolf T, Kiderlen T, Atta J, Stephan C, Kann G, Brodt H, et al. Successful treatment of AIDS-associated, primary CNS lymphoma with rituximab- and methotrexate-based chemotherapy and autologous stem cell transplantation. Infection. 2014;42:445-7.

100. Bohlius J, Schmidlin K, Costagliola D, Fätkenheuer G, May M, Caro Murillo AM, et al. Prognosis of HIV-associated nonHodgkin lymphoma in patients starting combination antiretroviral therapy. AIDS. 2009;23:2029-37.

101. Gopal S, Patel MR, Yanik EL, Cole SR, Achenbach CJ, Napravnik S, et al. Temporal trends in presentation and survival 
for HIV-associated lymphoma in the antiretroviral therapy era. J Natl Cancer Inst. 2013;105:1221-9.

102. Omuro AMP, Ben-Porat LS, Panageas KS, Kim AK, Correa $\mathrm{DD}$, Yahalom $\mathrm{J}$, et al. Delayed neurotoxicity in primary central nervous system lymphoma. Arch Neurol. 2005;62: 1595-600.

103. Correa DD, Maron L, Harder H, Klein M, Armstrong CL, Calabrese $\mathrm{P}$, et al. Cognitive functions in primary central nervous system lymphoma: literature review and assessment guidelines. Ann Oncol. 2007; 18:1145-51.

104. Correa DD, Shi W, Abrey LE, Deangelis LM, Omuro AM, Deutsch MB, et al. Cognitive functions in primary CNS lymphoma after single or combined modality regimens. Neuro Oncol. 2012;14:101-8.
105. Fossard G, Nicolas-Virelizier E, Rey P, Ducray F, Jouanneau E, Faurie P, et al. Utility of post therapy brain surveillance imaging in the detection of primary CNS lymphoma (PCNSL) relapse. Blood. $2013 ; 122$. Presented an important observation in a relatively large retrospective cohort that $80 \%$ of PCNSL relapses occur in between surveillance scans, thus demonstrating very limited utility for routine post-therapy imaging.

106. Nayak L, Hedvat C, Rosenblum MK, Abrey LE, De Angelis LM. Late relapse in primary central nervous system lymphoma: clonal persistence. Neuro Oncol. 2011;13:525-9.

107. Ferreri AJM, Ciceri F, Brandes AA, Montanari M, Balzarotti M, Spina M, et al. MATILDE chemotherapy regimen for primary CNS lymphoma: results at a median follow-up of 12 years. Neurology. 2014;82:1370-3. 